\title{
Mapeamento dos fluxos de informação e conhecimento: a governança de TI sob a ótica das redes sociais
}

\section{Mapping information and knowledge flow: IT governance from the perspective of social networks}

\author{
Renelson Ribeiro Sampaio' \\ Conrado Pereira Rosa ${ }^{2}$ \\ Hernane Borges de Barros Pereira ${ }^{1,3}$
}

\begin{abstract}
Resumo: Competitividade empresarial pressupõe a capacidade da empresa de definir e implantar estratégias concorrenciais que lhe permitam a sobrevivência e o desenvolvimento no longo prazo - em outras palavras, a sua perenidade. Dentre estas estratégias, a de inovação tecnológica associada com desenvolvimento da capacitação técnico-científica da organização e, portanto, dos indivíduos que atuam nos seus diversos processos, tem adquirido uma crescente importância no planejamento e nas ações empresariais. Neste contexto, as relações interpessoais constituem um fator crítico nos processos de inovação, os quais devem ser continuamente aperfeiçoados. O principal objetivo deste trabalho é contribuir na compreensão das dinâmicas associadas a estes processos a partir do estudo de caso desenvolvido com foco nos procedimentos e nos fluxos de informação, gerados pela Gerência de Capacidade de uma grande empresa de telecomunicação no Brasil. A pesquisa foi desenvolvida considerando as abordagens qualitativa e quantitativa. Por um lado, com base na pesquisa qualitativa foi possível estudar, a partir da perspectiva filosófica interpretativa, o fenômeno social relacionado com a difusão de conhecimentos entre membros de uma equipe responsável pelas ações e proposições técnicas na Gerência de Capacidade da empresa de telecom em questão. Por outro lado, a metodologia quantitativa permitiu a utilização de alguns modelos matemáticos de modo a mensurar propriedades (e.g. coesão, centralidade, prestígio e conectividade) das redes estudadas, assim como caracterizá-las topologicamente. O presente trabalho foi desenvolvido com base no modelo proposto pelos pesquisadores Nonaka e Takeuchi (1977) para analisar e descrever os processos de criação e de difusão do conhecimento na organização, associado com as técnicas de Análise de Rede Social (ARS). Os resultados sugerem que existem indícios das redes estudadas apresentarem a topologia livre de escala, o que torna os fluxos de informação e conhecimento resistentes a "ataques" aleatórios (e.g. saída espontânea de um membro da equipe), mas vulneráveis a "ataques" planejados (e.g. retirada de indivíduos bem conectados ou hubs).
\end{abstract}

Palavras-chave: Criação e difusão do conhecimento. Análise de rede social. Tecnologia da informação. ITIL.

\begin{abstract}
Business competitiveness requires the company's ability to define and implement competitive strategies that will allow its survival and development in the long term - in other words, its sustainability. Among these strategies, technological innovation associated with the development of the technical-scientific capacity of the company, and therefore, of the individuals who work in its various processes, has acquired a position of growing importance in corporate planning and actions. Accordingly, interpersonal relations are a critical factor for innovation processes, which must be continuously improved. The main objective of this study is to contribute to the understanding of the dynamics associated with these processes from the case study conducted focusing on procedures and information flows generated by the Capacity Management of a large telecommunications company in Brazil. The research was carried out based on qualitative and quantitative approaches. On the one hand, from the philosophical perspective of interpretation and based on the qualitative research, it was possible to study the social phenomenon related to the diffusion of knowledge among members of a team responsible for the actions and technical proposals in the capacity management of the telecom company being studied. On the other hand, the quantitative approach allowed the use of mathematical models in order to measure properties (e.g. cohesion, centrality, prestige and connectivity) of the networks studied and to provide their topological characterization. The present study was developed based
\end{abstract}

\footnotetext{
${ }^{1}$ Programa de Modelagem Computacional, Serviço Nacional de Aprendizagem Industrial - SENAI Cimatec, Av. Orlando Gomes, 1845, CEP 41650-010, Salvador, BA, Brasil, e-mail: renelson.sampaio@ fieb.org.br

${ }^{2}$ Consultor em Banco de Dados, Av. Padre Antonio José dos Santos, 530, Brooklin, CEP 04563001, São Paulo, SP, Brasil

${ }^{3}$ Departamento de Ciências Exatas, Universidade Estadual de Feira de Santana - UEFS, Campus Universitário,

Módulo 5, CEP 44031-460, Feira de Santana, BA, Brasil
}

Recebido em 24/8/2010 — Aceito em 12/4/2012

Suporte financeiro: Nenhum. 
on the model proposed by Nonaka and Takeuchi (1977) in order to analyze and describe the processes of creation and dissemination of knowledge in the organization, associated with the methods of Social Network Analysis (SNA). The results suggest that the networks studied exhibit scale-free topology, which makes the flow of information and knowledge resistant to random "attacks" (e.g. spontaneous resignation of a team member), but vulnerable to planned “attacks" (e.g. either reallocation or dismissal of well-connected individuals or hubs).

Keywords: Creation and diffusion of knowledge. Social network analysis. Information technology. ITIL.

\section{Introdução}

A dependência mútua entre a organização e a tecnologia da informação (TI) tem sido observada de forma crescente e explícita nos modelos de governança corporativa. Esta relação de interdependência é motivada principalmente pela necessidade de uma maior transparência na administração e controle dos recursos de TI, o que compreende um conjunto de aplicações, infraestrutura computacional e de comunicação de dados da organização. Este desafio tem proporcionado o desenvolvimento de modelos de governança nos quais a tecnologia da informação passa a fazer parte integrante da organização e de sua estratégia, buscando uma resposta à evolução e à complexidade da competição dos mercados no século XXI. O interesse pela governança corporativa não é novo, mas a necessidade de estabelecer e validar os elos de confiança com a sociedade, particularmente com os stakeholders, acelerou a evolução da governança nas organizações no que tange a TI. Observa-se que empresas que adotam e seguem as melhores práticas sugeridas pela governança chegam a ter aumentos na ordem de 10 a $12 \%$ no seu valor de mercado (WEILL; ROSS, 2006, p. 4)

Uma importante iniciativa para regulamentar a governança, quanto ao combate à aplicação fraudulenta da TI nos processos de gestão organizacional, foi proposta pelos senadores americanos Michael Oxley e Paul Sarbanes e aprovada em 2002 pelo governo americano para acabar com as fraudes contábeis, conhecida como lei Sarbanes-Oxley e difundida pelo acrônimo SOX, possui ampla aderência no mercado e interdependência com os modelos de governança de TI. Em uma pesquisa realizada com 245 executivos, foi observado que $51 \%$ destes acreditam que a implantação da SOX aumentou a confiança dos investidores na organização. Por outro lado, do total entrevistado, $70 \%$ dos executivos entenderam que os investimentos para aderência das organizações à SOX, retornarão ao negócio das organizações por meio do aumento de valor agregado (COMPUTERWORLD, 2007, p. 1).

Alinhar a TI com o negócio da organização equivale a ter uma estratégia coerente com a estratégia da própria organização (VALDEUSO, 2006, p. 35). Para Clean (2006, p. 63), a TI virá a se tornar um diferencial competitivo para a organização, quando as suas aplicações e soluções refletirem um adequado conhecimento dos problemas e do negócio da organização. A informação e a tecnologia que suportam o negócio da organização representam o ativo de maior valor agregado da organização (FAGUNDES, 2007, p. 1). O valor deste ativo é resultante da simbiose entre a organização, a TI e as pessoas. A evolução desta simbiose ocorre, dentre outros fatores, quando a governança de TI subsidia e/ou participa das tomadas de decisões pertinentes ao negócio da organização.

O objetivo de todo processo de governança de TI é estabelecer a infraestrutura adequada para que a organização possa prover um serviço de qualidade com respeito ao seu negócio. O uso da tecnologia por si só não é um diferencial capaz de proporcionar tal qualidade. A integração da TI ao negócio e o uso apropriado dos serviços resultantes desta integração em prol do negócio fim da organização é o diferencial.

Dentre as propostas para gerenciamento de serviços de TI, o conjunto de melhores práticas para a governança de TI, sugerido pela Biblioteca de Infraestrutura de TI (Information Technology Infrastructure Library - ITIL versão 2, marca registrada do Office of Government Commerce do Governo Inglês), fornece o ambiente relatado neste trabalho, da empresa selecionada para estudo de caso.

A ITIL representou uma evolução no tratamento dos processos operacionais relacionados à infraestrutura de TI, provendo as organizações de um referencial estruturado e funcional para lidar com a multiplicidade de ações e impactos transversais da TI. Entretanto, é importante destacar que as melhores práticas sugeridas pela ITIL são planejadas e executadas por pessoas. Assim sendo, tornou-se uma necessidade e, ao mesmo tempo, um desafio fazer o mapeamento das relações existentes com base nos fluxos de informação e conhecimento em uma organização no âmbito de TI. São essas relações em última análise que dão suporte à execução destes conjuntos de melhores práticas. Nesse sentido, propõe-se a aplicação das técnicas de análise de redes sociais como a mais adequada e natural para investigar tais relações.

Uma vez construída a rede social da organização ou, pelo menos, de um de seus setores, é possível avaliar o desempenho do fluxo de informação e conhecimento de cada indivíduo integrante na rede, possibilitando assim desenhar eventuais mecanismos necessários 
para potencializar o processo de socialização e consequentemente para a difusão do conhecimento.

O objetivo deste trabalho é mapear os fluxos de informação e conhecimento dentro da organização a partir das redes estabelecidas pelos indivíduos que pertencem à equipe da Gerência de Capacidade. A base teórica utilizada para a compreensão desses fluxos é o modelo de conversão de conhecimento proposto pelos pesquisadores Ikujiro Nonaka e Takeuchi (1997), doravante referenciado por modelo SECI. O Acrônimo SECI foi definido pelos mesmos autores em obra posteriormente lançada (NONAKA; TAKEUCHI, 2008), contudo tendo o mesmo significado da obra de 1997, sendo lido: por meio da Socialização, Externalização, Combinação e Internalização - SECI. Este modelo considera que a criação do conhecimento se inicia no indivíduo, sendo posteriormente difundido e desenvolvido na organização por meio das relações entre os demais indivíduos que a compõem. Assim, o desafio da organização consiste em proporcionar um ambiente em que as relações interpessoais sejam um elemento-chave de sucesso nos processos de socialização do conhecimento. A análise de rede social, utilizada como uma ferramenta para mapear os fluxos de informação e conhecimento, permitirá identificar de que forma uma relação entre membros de uma rede pode fornecer um canal de comunicação para estes fluxos.

A organização deste trabalho se dá com a seguinte estrutura: a seção 2 estabelece um paralelo entre o Conhecimento Organizacional fundamentada no modelo SECI; a seção 3 apresenta brevemente aspectos históricos e características de redes sociais importantes para a análise proposta; a seção 4 relata o estudo de caso trabalhado; e, finalmente, a seção 5 apresenta as considerações finais.

\section{Conhecimento organizacional}

A premissa básica adotada neste trabalho é que não se gerencia a criação e difusão do conhecimento, senão a adoção de práticas e processos de negócio que consolidam as condições favoráveis no ambiente da organização em prol do conhecimento individual e organizacional (VON KROGH et al., 2001, p. 12).

O conhecimento, ao contrário da informação, diz respeito a crenças e compromissos, sendo uma função direta de uma atitude, perspectiva ou intenção específica, i.e., sempre será o conhecimento 'com algum fim'. Por outro lado, a aplicabilidade da informação é uma das características do conhecimento organizacional. O valor do conhecimento oriundo da organização deve ser agregado ao negócio, pois a visão cartesiana e ocidental sobre uma organização prepara-a para, simplesmente, processar informação tendenciosamente de origem externa. Embora neste modelo existam níveis de eficácia baixos de produção de conhecimento interno e altos de aquisição de conhecimento externo, mesmo que de forma subjetiva e imprecisa, a possibilidade de um balanceamento entre informação/conhecimento externos e internos à organização tende a fornecer melhores resultados (NONAKA; TAKEUCHI, 1997, p. 64). A formação de um ciclo virtuoso e positivo entre a criação e difusão do conhecimento, a inovação contínua e a vantagem competitiva tendem a ser uma trilha de sucesso no caminho das organizações (DAVENPORT; PRUSAK, 1998, p. 18; NONAKA; TAKEUCHI, 1997, p. 274).

Nonaka e Takeuchi (1997, p. 1) definem a criação do conhecimento empresarial como a capacidade de uma empresa criar novos conhecimentos, difundi-los e incorporá-los a produtos, serviços e sistemas. A grande contribuição desses autores reside na compreensão de que os processos de criação do conhecimento não decorrem simplesmente de procedimentos pré-definidos, formais ou automáticos da organização. Ao contrário, é o resultado de um processo essencialmente humano, por natureza complexo e dinâmico. Os autores argumentam com base no trabalho de Michael Polanyi (POLYANI, 2009), que o conhecimento possui duas categorias: a tácita e a explícita. A criação do conhecimento ocorre por meio de um processo contínuo de conversão do conhecimento tácito para o conhecimento explícito e vice-versa. Basicamente ocorrem quatro modos de conversão: socialização, Externalização, combinação e Internalização (NONAKA; TAKEUCHI, 1997, p. 65-69). Estes compõem o fundamento da criação do conhecimento no modelo SECI.

A incorporação de experiências individuais ao conhecimento organizacional é difícil, principalmente quando envolve fatores intangíveis (e.g. palpites subjetivos, insights, conclusões, crenças, valores, perspectivas, ideias, emoções, imagens, símbolos, significados e significantes ou, resumidamente, os elementos cognitivos). Entretanto, seu êxito permite que este conhecimento, chamado tácito, agregue valor à organização. Um dos papéis da organização é, portanto, promover um ambiente propício para a conversão do conhecimento tácito em explícito (NONAKA; TAKEUCHI, 1997).

No contexto da organização, a criação do conhecimento ocorre em três níveis: do indivíduo, do grupo e da própria organização (intraorganizacional). Cada um desses níveis ocupa o seu papel de importância no modelo SECI. A criação é papel do indivíduo. É o indivíduo que cria e difunde o conhecimento. As relações estabelecidas entre os indivíduos formam o grupo, no qual se identificam as primeiras ocorrências de difusão do conhecimento. A organização estabelece o contexto (infraestrutura, normas, padrões, cultura) em que os grupos existentes interagem. A interação pode também ocorrer no nível interorganizacional (BASTOS; SANTOS, 2007, p. 30). 
A Tabela 1 estabelece, com base nos trabalhos desenvolvidos por Nonaka e Takeuchi (1997) e, posteriormente, por Von Krogh, Ichijo e Nonaka (2001), uma integração entre as fases do processo de criação do conhecimento, as condições capacitadoras e os modos de conversão de conhecimento do modelo SECI. Na coluna 'Condições Capacitadoras para criação de conhecimento', os itens em negrito indicam as condições relacionadas aos modos de conversão destacados na coluna 'Modos predominantes de conversão do conhecimento'.

Visivelmente, na fase de difusão do conhecimento, os quatro modos de conversão (Socialização, Externalização, Internalização, combinação) estão presentes e são necessários. Dentre os quatro modos de conversão do conhecimento, a socialização fornece uma fonte constante de difusão do conhecimento. Avaliar a socialização permitiria, portanto inferir a respeito da difusão do conhecimento. É neste sentido que se utiliza a Análise de Redes Social (ARS), uma vez que a ARS incorpora a capacidade de aferir ou mapear as relações interpessoais da organização de forma a criar uma fotografia da socialização existente.

De um modo geral, a informação pode ser considerada sob dois pontos de vista: a informação "sintática", ou o volume de informações, e a informação "semântica", ou o significado. A informação semântica agrega valor à criação e difusão do conhecimento; A informação semântica em abundância no conhecimento tácito é a maior fonte de criação do conhecimento organizacional (NONAKA; TAKEUCHI, 1997, p. 64). Um dos focos dos modelos de governança em TI está na documentação da informação sintática controlada e acompanhada por ferramentas sugeridas na implantação de cada modelo de governança.

A utilização dos modos de conversão é uma ferramenta poderosa, quando envolve condições capacitadoras do conhecimento integradas ao conceito de solicitude organizacional, proposto por Von Krogh, Ichijo e Nonaka (2001). Na visão desses autores, tanto a criação quanto a difusão do conhecimento exigem muitos relacionamentos na organização.

Tabela 1. Integração de processos \& conceitos do modelo SECI.

\begin{tabular}{|c|c|c|c|c|c|c|}
\hline $\begin{array}{c}\text { Fases do processo de criação } \\
\text { do conhecimento }\end{array}$ & Visão macro & $\begin{array}{c}\text { Condições capacitadoras } \\
\text { para criação de } \\
\text { conhecimento }\end{array}$ & \multicolumn{4}{|c|}{$\begin{array}{c}\text { Modos } \\
\text { predominantes } \\
\text { de conversão do } \\
\text { conhecimento }\end{array}$} \\
\hline $\begin{array}{l}\text { Compartilhamento do } \\
\text { conhecimento tácito }\end{array}$ & $\begin{array}{l}\text { O conhecimento tácito mantido } \\
\text { pelos indivíduos é a base da } \\
\text { criação do conhecimento. }\end{array}$ & $\begin{array}{l}\text { Intenção } \\
\text { Autonomia } \\
\text { Flutuação e caos criativo } \\
\text { Redundância } \\
\text { Variedade de requisitos }\end{array}$ & : & 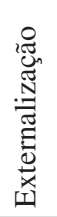 & 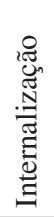 & 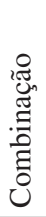 \\
\hline $\begin{array}{l}\text { Criação de } \\
\text { Conceitos }\end{array}$ & $\begin{array}{l}\text { O modelo mental tácito } \\
\text { compartilhado é verbalizado } \\
\text { em palavras frases e finalmente } \\
\text { em conceitos explícitos. }\end{array}$ & $\begin{array}{l}\text { Intenção } \\
\text { Autonomia } \\
\text { Flutuação e caos criativo } \\
\text { Redundância } \\
\text { Variedade de requisitos }\end{array}$ & 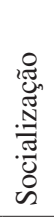 & : & 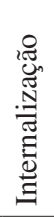 & 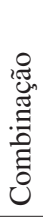 \\
\hline $\begin{array}{l}\text { Justificação de } \\
\text { Conceitos }\end{array}$ & $\begin{array}{l}\text { Novos conceitos criados por } \\
\text { indivíduos ou equipe precisam } \\
\text { ser justificados em algum } \\
\text { momento do procedimento. }\end{array}$ & $\begin{array}{l}\text { Intenção } \\
\text { Autonomia } \\
\text { Flutuação e caos criativo } \\
\text { Redundância } \\
\text { Variedade de requisitos }\end{array}$ & 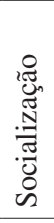 & 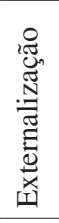 & 预 & 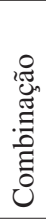 \\
\hline Construção de um arquétipo & $\begin{array}{c}\text { O conceito justificado é } \\
\text { transformado em algo tangível } \\
\text { ou concreto. }\end{array}$ & $\begin{array}{l}\text { Intenção } \\
\text { Autonomia } \\
\text { Flutuação e caos criativo } \\
\text { Redundância } \\
\text { Variedade de requisitos }\end{array}$ & 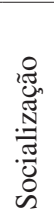 & 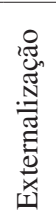 & 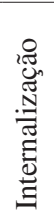 & 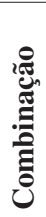 \\
\hline $\begin{array}{l}\text { Difusão interativa do } \\
\text { conhecimento }\end{array}$ & $\begin{array}{c}\text { A criação do conhecimento } \\
\text { organizacional é um processo } \\
\text { interminável que se atualiza } \\
\text { continuamente. }\end{array}$ & $\begin{array}{l}\text { Intenção } \\
\text { Autonomia } \\
\text { Flutuação e caos criativo } \\
\text { Redundância } \\
\text { Variedade de requisitos }\end{array}$ & 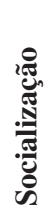 & : & 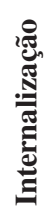 & نْ \\
\hline
\end{tabular}

Fonte: Autores, baseado em Nonaka e Takeuchi (1997). 
Para que o conhecimento pessoal seja efetivamente compartilhado, os indivíduos devem confiar que os outros estejam dispostos a ouvir e reagir às suas ideias. Esta disposição é denominada de solicitude organizacional. De fato, ainda de acordo com os autores citados, o clima organizacional é uma das condições mais importantes: "Constatamos fortes indícios de que o clima organizacional que fomenta a confiança, a solidariedade e as redes pessoais entre os empregados é uma das condições mais importantes para a difusão eficaz do conhecimento técnico e administrativo." (VON KROGH; ICHIJO; NONAKA, 2001, p. 41).

\section{Análise de redes sociais}

As redes sociais passam a ganhar força como objeto de pesquisa quando, em 1937, o psicoterapeuta Jacob Levy Moreno propõe o conceito da sociometria, visando explorar os vínculos estabelecidos entre indivíduos em um grupo de uma dada organização, e os sociogramas, que são representações gráficas dessas relações (MORENO, 1937; WASSERMAN; FAUST, 1999). Ao final dos anos 1930, três correntes destacavam-se nesta linha de pesquisa: (i) pesquisadores de Havard estudavam modelos de relações interpessoais e a formação de cliques; (ii) analistas sociométricos que estudavam pequenos grupos; e (iii) antropólogos de Manchester cujas pesquisas concentravam-se na estrutura das relações de comunidades em sociedades tribais e aldeãs (LÓPEZ; SCOTT, 2000). Entre as décadas dos anos 1960 e 1970, passamos a ter uma Análise de Redes Sociais contemporânea com a fusão das três correntes e a junção com outras pesquisas.

O referencial teórico utilizado nesta pesquisa para análise de redes foi embasado em grande parte segundo os conceitos propostos por Wasserman e Faust (1999). A perspectiva da rede social foca os relacionamentos entre entidades sociais, por exemplo, a comunicação entre membros de um grupo, transações econômicas entre corporações e comércio ou tratado entre nações. O foco nos relacionamentos é uma adição importante para a pesquisa de padrão social e comportamental.

Análise de Redes utiliza análises numéricas e estatísticas necessárias para o estudo de relações entre entidades e são aplicáveis a muitas áreas do conhecimento. Dentre elas: a Sociologia, a Antropologia, a Psicologia, a Economia, a Física, a Computação e a Ciência da Informação (MATHEUS, 2005; MATHEUS; SILVA, 2006).

Há vários conceitos na Análise de Redes que embasam as discussões de redes sociais (WASSERMAN; FAUST, 1999). Tais como: ator, ligações relacionais, díade, tríade, subgrupo, grupo, relação e rede. Desta forma, a Análise de Redes Sociais fornece uma maneira precisa de definir conceitos sociais importantes, uma alternativa teórica à suposição de atores sociais independentes, e um framework para teorias de teste sobre relacionamentos sociais estruturados (WASSERMAN; FAUST, 1999).

O foco do estudo da Análise de Redes Sociais é sobre os atores e as relações (laços) por eles mantidos socialmente em forma de elos, na rede da organização. Marteleto (2001) considera que as redes sociais ou networks são representações de um conjunto de participantes autônomos, unindo ideias e recursos em torno de valores e interesses compartilhados.

As redes sociais são representadas matematicamente como grafos, i.e. estruturas formadas por vértices (ou nós) que são os atores, conectados por arestas que correspondem aos laços relacionais entre os atores. A direção do fluxo é representada pela seta nas conexões entre os vértices, indicando a origem da informação e conhecimento.

O complemento da ARS aos modelos de governança de TI, em específico neste trabalho, está na capacidade de mapear as relações interpessoais, fornecendo ao modelo SECI uma maneira de aferir a condição atual da organização quanto ao fluxo de informação e conhecimento.

No modelo SECI, a dimensão ontológica estabelece um elo forte entre o indivíduo e o seu grupo, ao estabelecer que a difusão do conhecimento dependa fundamentalmente das relações existentes entre os integrantes do grupo. A ARS fornece o ferramental não disponibilizado tanto no modelo SECI quanto na ITIL para aferir tais relações. Com o uso da ARS, foi possível observar que existe uma dificuldade em mapear as relações na mesma velocidade e dinamismo que estas ocorrem. Em outras palavras, a ARS permite mapear as relações em determinados instantes e não de forma contínua, ou seja, a ARS é uma ferramenta capaz de aferir estas relações, ainda que seja por meio de uma sequência de eventos discretos no tempo, como uma coleção de fotografias. Mas, mesmo sendo uma extração discreta de um processo dinâmico, os resultados obtidos em diferentes momentos contribuíram significativamente para a análise.

\section{Estudo de caso}

A rede social estudada é formada pelos atores que compõem a Gerência de Capacidade da empresa VIVO S.A., selecionada neste estudo de caso, que atua no setor de telecomunicações no Brasil e possui implementada a ITIL versão 2 no ambiente de TI. Dentre as empresas de projeção nacional, esta registra um elevado grau de adoção das gerências sugeridas nas melhores práticas aderentes à ITIL, tendo neste ambiente a Gerência de Capacidade formalmente constituída e estruturada nos fluxos processuais preconizados pelo ITIL entre as demais gerências.

O questionário utilizado para a consecução dos objetivos deste trabalho foi dividido em três blocos. 
No primeiro bloco (dados básicos), são levantadas questões individuais referentes à formação profissional, gênero, tempo de trabalho, setor e contato. Já no segundo bloco (visão sobre processos), obtêm-se informação sobre os processos da área de TI baseada na ITIL, não sendo objeto do questionário os itens administrativos da organização. O último bloco (visão sobre o relacionamento) está subdividido em duas partes: a primeira busca identificar as fontes de informação e a segunda trata da obtenção/construção do Conhecimento no âmbito organizacional. Em cada um dos segmentos técnico da área de TI (i.e. banco de dados, rede, sistema operacional, storage, $w e b$ e aplicação), foram analisadas a prioridade, a frequência de utilização e a intensidade necessária para a solução de um determinado processo/problema com respeito às fontes de informação e conhecimento identificadas.

O questionário aplicado procurou registrar dos atores que fizeram parte da pesquisa a percepção individual sobre os processos nos quais atuam na organização, os relacionamentos interpessoais e as suas fontes de conhecimento.

Foram estabelecidos dois critérios para a definição dos atores, tendo em vista a necessidade de mapear os elos das cadeias de fluxo de informação e conhecimento: o primeiro critério usado na interação inicial está em que o ator deve fazer parte da Gerência de Capacidade da empresa pesquisada; o segundo critério está baseado no resultado da questão onze do questionário ('Elencar até 10 pessoas, da sua área ou não, as quais você considera as suas fontes de consulta para obtenção de informações na organização.'), que forneceu a segunda interação.

Com base nas respostas obtidas dos 11 membros da equipe da Gerência de Capacidade da empresa, foi possível determinar mais 43 atores, perfazendo o total de 54 questionários aplicados. Dos 43 atores apontados pela equipe de capacidade como as suas fontes de informação na organização, 34 responderam e 9 não retornaram. De um total de 54 questionários, foi obtido um retorno de $81 \%$. No momento da pesquisa (outubro de 2008), a empresa tinha 290 colaboradores associados à diretoria de infraestrutura de operações de sistemas. Os dados aqui analisados preservam o nome dos atores envolvidos bem como informações confidenciais para a organização, nos termos estabelecidos no contrato de confidencialidade assinado entre as partes.

O conceito de problema operacional, utilizado neste trabalho, é definido quando um item técnico do ambiente de TI é afetado (e.g. um problema em um hardware ou no software que gerencia tal hardware, tendo variações de fornecedor, sistema, versões, etc.). Neste caso, o conhecimento técnico especializado, seja explícito ou tácito, é estritamente necessário para a solução.
Especificamente, das 17 questões do questionário, as de número $9,11,12,13,14$ e 15 focalizaram a conversão do conhecimento tácito em explícito. Seus objetivos estão expostos na Tabela 2. As demais perguntas permitem a descrição de aspectos qualitativo e quantitativo da Gerência de Capacidade.

Quando os atores da equipe de capacidade foram questionados sobre as fontes de informação e conhecimento utilizadas em situações de problemas operacionais classificados por grau de complexidade, grau de urgência e grau de impacto, todos sobre o negócio-fim da organização, observaram que: (1) a principal fonte de conhecimento é o indivíduo e (2) as redes mapeadas foram alteradas de acordo com o tipo de problema operacional e seu grau correspondente.

$\mathrm{Na}$ pesquisa, foram considerados seis tipos de problemas operacionais, usando como critério para a sua definição segmentos técnicos da área de TI (i.e. banco de dados, rede, sistema operacional, storage, web e aplicação). Cada tipo de problema supracitado é classificado de acordo com o seu grau de complexidade (i.e. tipo de problema que abrange muito elementos técnicos, que possuem ou não relação entre si), urgência (i.e. tipo de problema que requer ação imediata, não admitindo delongas na solução técnica) e impacto (i.e. permite avaliar o impacto de um dado problema técnico no negócio-fim da organização) quanto ao negócio-fim da organização e usa a escala: Baixa, Média Baixa, Média Alta, Alta. Deve-se destacar que os segmentos técnicos supracitados independem do modelo de melhores práticas de governança de TI a ser adotado pela organização. No presente estudo, estes segmentos técnicos são de vital importância para o negócio-fim da organização estudada, uma vez que toda atividade e a própria estratégia de negócio de uma empresa de telecomunicação está baseada na tecnologia de informação. Seu sucesso depende, em grande medida, na capacidade em responder aos desafios tecnológicos associados à TI.

Nas redes mapeadas, observa-se a variação dos atores no que tange ao tipo do problema (Figura 1). As Figuras 1a, 1b e 1c apresentam as redes dos fluxos de informação e conhecimento para todos os problemas operacionais, considerando respectivamente os graus de complexidade, urgência e impacto. $\mathrm{Na}$ pesquisa realizada, foram identificadas todas as redes associadas com cada um dos problemas operacionais listados acima. Entretanto, para efeito de ilustração do argumento neste trabalho, optou-se por apresentar a rede formada por todos os problemas.

Observa-se, na Figura 1, que as relações entre os atores mudam de acordo com as situações provenientes de problemas operacionais classificadas pelos graus de complexidade, urgência e impacto. Essas mudanças revelam uma falta de aderência 
Tabela 2. Descrição e objetivos das questões 9, 11, 12, 13, 14 e 15.

\begin{tabular}{|c|c|}
\hline Questões & Objetivo principal \\
\hline $\begin{array}{l}\text { 09) Em uma dúvida processual ocorrida no seu dia a dia de } \\
\text { trabalho a sua primeira fonte de pesquisa está na ferramenta } \\
\text { oficial da organização ou em um colaborador? }\end{array}$ & $\begin{array}{l}\text { - Identificar a primeira fonte de pesquisa usada } \\
\text { para resolver problemas relacionados aos } \\
\text { fluxos processuais da organização. }\end{array}$ \\
\hline $\begin{array}{l}\text { 11) Elencar até } 10 \text { pessoas, da sua área ou não, as quais } \\
\text { você considera as suas fontes de consulta para obtenção de } \\
\text { informações na organização. Use as escalas propostas na página } \\
\text { acima (consulte as tabelas auxiliares abaixo) para determinar a } \\
\text { prioridade, a frequência e a intensidade, lembrando que estas } \\
\text { podem se repetir. }\end{array}$ & $\begin{array}{l}\text { - Mapear a rede interpessoal de relacionamento } \\
\text { do indivíduo, estando esta associada ou não à } \\
\text { rede formal delimitada pelos processos. }\end{array}$ \\
\hline $\begin{array}{l}\text { 12) Em uma situação que exige prioridade máxima de solução, } \\
\text { qual é a sua primeira opção de fonte de informação a ser usada? }\end{array}$ & $\begin{array}{l}\text { - Entender o principal recurso utilizado pelo } \\
\text { indivíduo no momento em que este é cobrado } \\
\text { pela resolução de um determinado problema. }\end{array}$ \\
\hline $\begin{array}{l}\text { 13) Cruzando os Tipos de Problemas Operacionais com o } \\
\text { seu Grau de Complexidade médio da tecnologia e as Fontes } \\
\text { de Conhecimento descritas acima, preencha a tabela abaixo, } \\
\text { relacionando, para cada tipo de problema operacional, o seu } \\
\text { grau médio de complexidade e a sua Fonte de Conhecimento } \\
\text { prioritária para a solução/tratamento de cada um dos cinco tipos } \\
\text { de Problemas Operacionais. }\end{array}$ & \multirow{3}{*}{$\begin{array}{l}\text { - Associar a rede interpessoal à resolução } \\
\text { de problemas técnicos da área de TI. Esta } \\
\text { resolução necessita de conhecimento dos } \\
\text { processos organizacionais, do conhecimento } \\
\text { tácito necessário e principalmente um } \\
\text { conhecimento relacional capaz de mobilizar as } \\
\text { pessoas, a depender do grau de complexidade, } \\
\text { impacto e urgência relacionado à frequência, } \\
\text { intensidade e prioridade das relações. }\end{array}$} \\
\hline $\begin{array}{l}\text { 14) Cruzando os Tipos de Problemas Operacionais com o seu } \\
\text { Grau de Urgência médio para a solução do problema e as Fontes } \\
\text { de Conhecimento descritas acima, preencha a tabela abaixo, } \\
\text { relacionando para cada tipo de problema operacional, o seu grau } \\
\text { médio de urgência e a sua Fontes de Conhecimento prioritária } \\
\text { para a solução/tratamento de cada um dos cinco tipos de } \\
\text { Problemas Operacionais. }\end{array}$ & \\
\hline $\begin{array}{l}\text { 15) Cruzando os Tipos de Problemas Operacionais com o seu } \\
\text { Grau de Impacto médio que o problema gera no negócio-fim } \\
\text { da organização e as Fontes de Conhecimento descritas acima, } \\
\text { preencha a tabela abaixo, relacionando, para cada tipo de } \\
\text { problema operacional, o seu grau médio de impacto e a sua } \\
\text { Fonte de Conhecimento prioritária para a solução/ tratamento de } \\
\text { cada um dos cinco tipos de Problemas Operacionais. }\end{array}$ & \\
\hline
\end{tabular}

Fonte: autores.

entre aquilo de está preconizado pela ITIL (i.e. um conjunto de recomendações em prol da governança de TI) e o modus operandi observado. Assim, essas percepções, reveladas a partir das redes sociais, permitirão a realização de ajustes necessários nos seus procedimentos que devem ser incorporados às práticas da governança.

Outra análise realizada refere-se a fontes de informação consultadas, especialmente aquelas relacionadas ao conhecimento tácito. Para tanto, foram construídas duas redes sociais: a primeira (Figura 2) com base exclusivamente na Questão 11 (vide Tabela 2), a segunda (Figura 3) com base nas Questões 9, 12, 13, 14, 15 e 17.

As redes sociais construídas (Figuras 2 e 3 ) que dão a ideia de totalidade dos fluxos de informação e conhecimento da Gerência de Capacidade reforçam a necessidade de realização de ajustes necessários às práticas da governança. Considerando que as redes apresentadas nas Figuras 2 e 3 representam o meio em que ocorrem os fluxos de informação e conhecimento, buscou-se estudar as propriedades dessas redes a partir de alguns índices da ARS e da teoria das redes complexas. Os índices usados neste trabalho foram: o caminho mínimo médio $(L)$, o coeficiente de aglomeração médio $(C)$, a distribuição de graus das redes $(P(k))$ e o diâmetro das redes $(D)$, além da quantidade de vértices $(N)$ e arestas $(M)$ e do grau médio $(<k>)$. Esses índices são apresentados e discutidos por diversos autores (WATTS; STROGATZ, 1998; WASSERMAN; FAUST, 1999; ALBERT; BARABÁSI, 2002; NEWMAN, 2003; BOCCALETTI et al., 2006; COSTA et al., 2007;). Assim, o detalhamento de cada um dos índices supracitados está fora do escopo deste artigo. Não obstante, fazem-se necessárias as formalizações do caminho mínimo médio $(L)$ e do coeficiente de aglomeração médio $(C)$. 


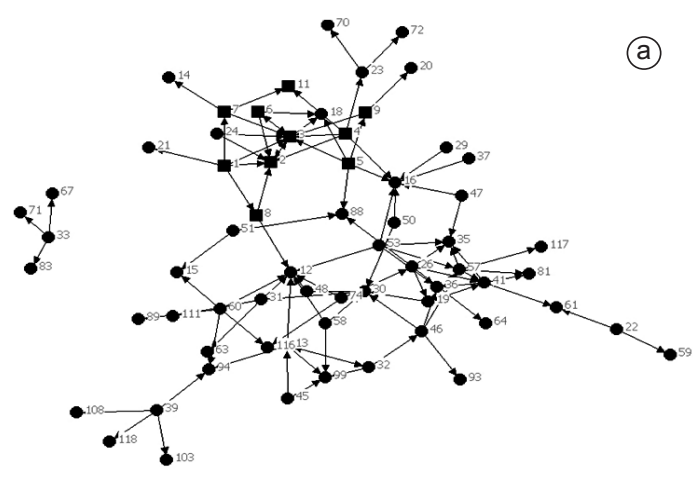

Grau de complexidade

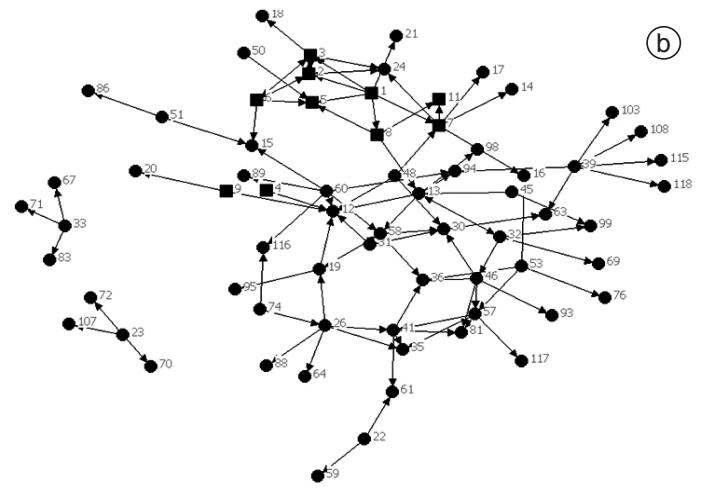

Grau de urgência

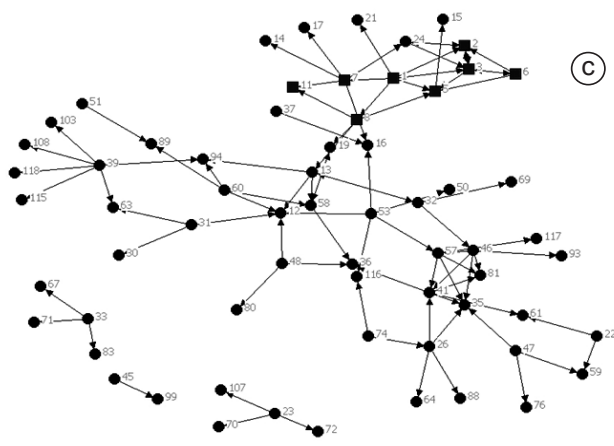

Grau de impacto

Figura 1. Rede para a Complexidade, Urgência e Impacto para todos os problemas operacionais. Os vértices quadrados representam os atores da equipe de capacidade; enquanto que os vértices redondos representam atores não pertencentes à equipe de capacidade. Os arcos que conectam os vértices indicam a direção da demanda de membros atores da equipe por informações de acordo com suas fontes prioritárias.

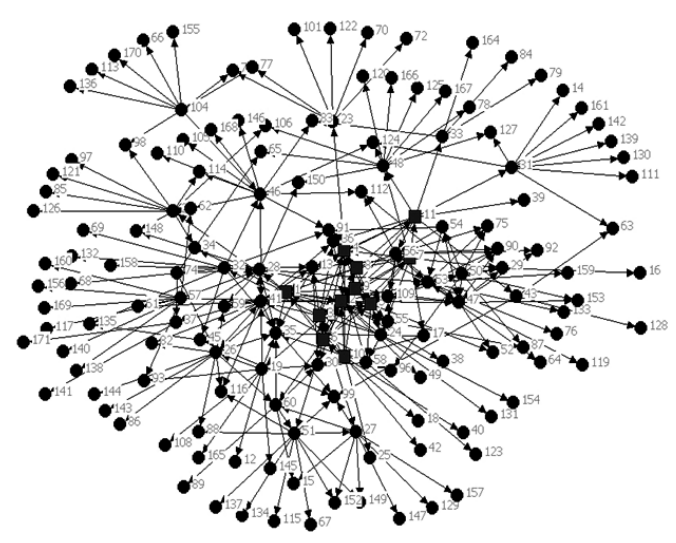

Figura 2. Rede com base na Questão 11. Os vértices quadrados representam os atores da equipe de capacidade; enquanto que os vértices redondos representam atores não pertencentes à equipe de capacidade. Os arcos que conectam os vértices indicam a direção da demanda de membros atores da equipe por informações de acordo com suas fontes prioritárias.

A média dos coeficientes de aglomeração dos vértices da rede é dada por $C=\frac{1}{N} \sum_{i=1}^{N} C_{i} \cdot \mathrm{O}$ coeficiente

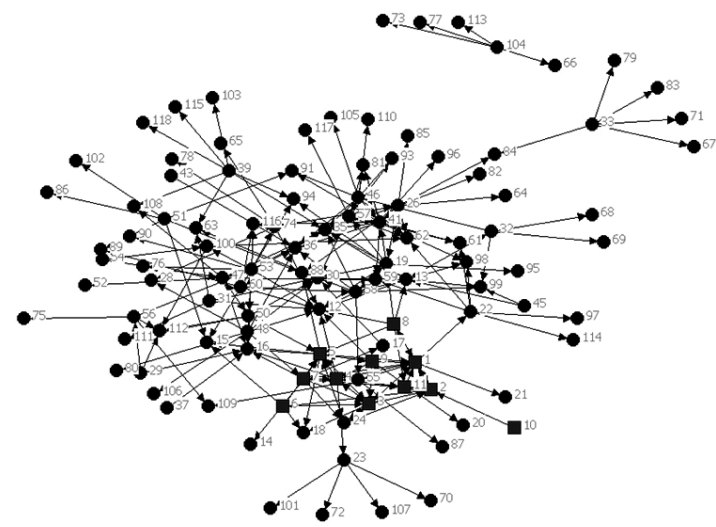

Figura 3. Rede com base nas Questões 9, 12, 13, 14, 15 e 17. Os vértices quadrados representam os atores da equipe de capacidade, enquanto que os vértices redondos representam atores não pertencentes à equipe de capacidade. Os arcos que conectam os vértices indicam a direção da demanda de membros atores da equipe por informações de acordo com suas fontes prioritárias.

de aglomeração de um vértice $i$, denotado por $C_{i}$ mede a proporção de arestas existentes entre os vizinhos do vértice $i$ que estão conectados e a quantidade 
Tabela 3. Comparação entre os índices de ARS.

\begin{tabular}{lccccccc}
\hline \multicolumn{1}{c}{ Redes questões } & $\mathbf{N}$ & $\mathbf{M}$ & $<\mathbf{k}>$ & $\Delta$ & $\mathbf{L}$ & $\mathbf{C}$ & $\mathbf{D}$ \\
\hline $\begin{array}{l}\text { Rede com base nas questões 09, 12, 13, } \\
\text { 14, 15 e 17 }\end{array}$ & 118 & 213 & 3,610 & 0,030 & 3,65 & 0,22 & 8 \\
Rede com base na questão 11 & 171 & 295 & 3,450 & 0,020 & 3,71 & 0,25 & 6 \\
\hline
\end{tabular}

Fonte: autores.

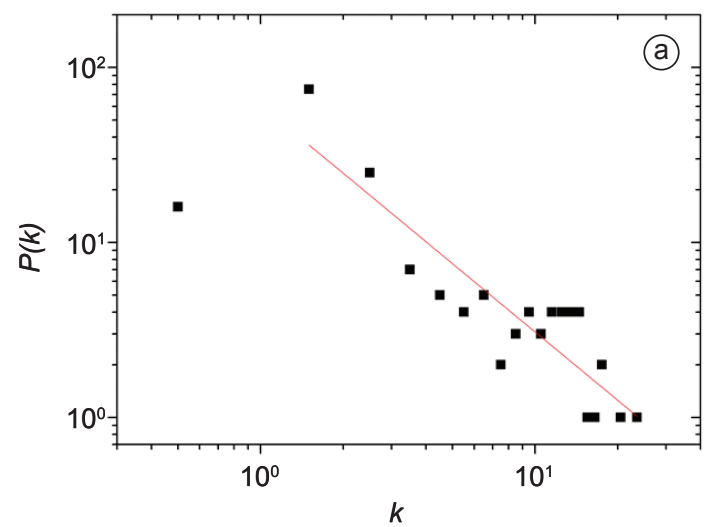

Distribuição de graus da rede com base na Questão 11. O valor do $\gamma=-1,2978$ e o ajuste $R^{2}=0,77997$

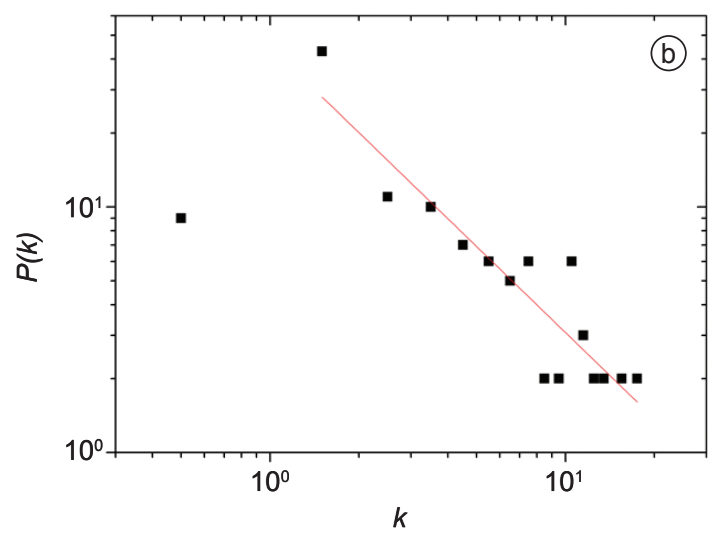

Distribuição de graus da rede com base nas Questões 09, 12, 13, 14, 15 e 17. O valor do $\gamma=-1,16386$ e o ajuste $R^{2}=0,8365$

Figura 4. Distribuição de graus das redes mapeadas plotadas em log-log, segundo a probabilidade $P(k) \sim k^{-\gamma}$.

máxima possível de arestas (WATTS; STROGATZ, 1998) (Equação 1):

$$
C_{i}=\frac{2 M_{i}}{k_{i}\left(k_{i}-1\right)}
$$

em que $M_{i}$ é a quantidade de arestas que conectam os vizinhos do vértice $i$ e $K_{i}$ é o grau do vértice $i$ (i.e. o número de arestas incidentes no vértice $i$ ). $\mathrm{O}$ coeficiente de aglomeração pode ter valores entre zero e um $(0 \leq C \leq 1)$. Em outras palavras, esse índice mostra até que ponto os amigos de um indivíduo, são amigos entre si.

O caminho mínimo médio é a distância geodésica média calculada a partir dos caminhos mínimos entre dois vértices de uma rede conectada (Equação 2):

$$
L=\frac{1}{N(N-1)} \sum_{i \neq j} d_{i j}
$$

em que $d_{i j}$ é a distância geodésica, em termos de quantidade de arestas, entre os vértices $i$ e $j$.

Os resultados para os índices $\langle k\rangle, \Delta, L, C$, e $D$ são mostrados na Tabela 3. É possível observar que, para esses índices, as redes estudadas apresentam valores muito próximos, podendo-se inferir que são similares quanto aos fluxos de informação e conhecimento. Observa-se também que os indivíduos dessas redes necessitam em média de, no máximo, 4 colegas para que as informações percorram toda a rede. Os valores dos coeficientes de aglomeração médios são baixos, o que indica a baixa conectividade entre os indivíduos das redes e, por conseguinte, a preferência da maioria dos indivíduos por determinados indivíduos de referência.

Calculando-se a distribuição de graus para ambas as redes apresentadas nas Figuras 2 e 3, cujas distribuições são apresentadas na Figura 4, observamos que há indícios de elas possuem características próximas às redes livres de escala. Considera-se o artigo de Barabási e Albert (1999) como marco dessa topologia (i.e. redes livres de escala). A distribuição de graus das redes mapeadas em escala log-log sugere uma lei de potência dada pela distribuição $P(k) \sim k^{-\gamma}$. Ressalva-se que, apesar dos ajustes não serem tão bons, é possível observar a existência de poucos hubs (i.e. atores nas redes que possuem muitas relações com outros atores) e de muitos atores com poucas conexões.

Do ponto de vista da topologia das redes, uma rede livre de escala possui um número relativamente pequeno de vértices que apresentam um grande número de conexões a outros nós, denominados hubs ou conectores. Os hubs determinam significativamente o grau de estabilidade de todo o conjunto das conexões da rede. No caso das redes estudadas, as constatações mostram que apenas alguns atores são preeminentes quanto aos fluxos de informação e conhecimento. Esta é uma situação que apresenta oportunidades de melhorias quando se atua em processos de alta 
complexidade que demandam respostas rápidas, situação observada pela organização e trabalhada em momento posterior à pesquisa.

\section{Considerações finais}

O papel desempenhado pela ITIL no contexto tecnológico é fundamental por uma questão mercadológica que permite aumentar o grau de confiança do investidor na organização. Ademais, a importância da ITIL deve-se principalmente pela capacidade de tornar os fluxos processuais sistêmicos e alinhados à organização. Tal situação permite criar a rede real dos fluxos de informação e conhecimento que possui derivações para as redes extraoficiais e está aderente ao que está preconizado pelo ITIL.

$O$ fato de o tempo não ser computado no modelo SECI de forma explícita, isto é, não ser uma variável pertencente ao modelo, limita e restringe as análises deste modelo. Em outras palavras, o modelo não permite prever o comportamento dinâmico das relações interpessoais. A ARS é um recurso pelo qual é possível estabelecer uma ideia da dinâmica dessas relações por meio da coleção de estados da rede ao longo de um determinado período, permitindo ajustes na rede que facilitem a difusão do conhecimento.

Assim, a aplicação das técnicas de ARS sob a óptica do modelo SECI de forma periódica possibilitaria uma visão continuada das relações, por meio de uma sequência de eventos discretos. Apesar de a ARS permitir visões das relações interpessoais em um dado instante no tempo, seu uso não reduz a importância de sua utilização no modelo SECI. A ARS permite, portanto, incorporar certo grau de dinamismo quando associada ao processo de mapeamento das relações intraorganizacionais, considerando a limitação exposta.

Estudar a informação por meio das ARS significa considerar as relações de poder que advêm de uma organização não hierárquica e espontânea, bem como a dinâmica do conhecimento e da informação que interfere nestas relações (MARTELETO, 2001, p. 73). Como já abordado, a estrutura hierárquica, combatida no modelo SECI, não favorece a criação e difusão do conhecimento. A ARS, por sua vez, permite explicitar os meios pelos quais os fluxos de informação e o conhecimento organizacional ocorrem independentemente da estrutura hierárquica da organização, em que os saberes tácitos e explícitos são potencializados pela capacidade dos atores de se relacionarem no meio intraorganizacional.

Considerando as redes estudadas, observamos que há indícios de elas possuem características próximas às redes livres de escala, por apresentar uma distribuição que segue uma lei de potência. Isto significa que, do ponto de vista dos fluxos de informação e conhecimento, há uma grande dependência da rede estudada com respeito aos hubs, tendo assim uma significativa concentração do conhecimento nestes vértices determinados (i.e. indivíduos da Gerência de Capacidade). Tal característica pode ser vista como uma oportunidade de melhoria quando observada sob a óptica do modelo SECI, especialmente quando se observa a fase de socialização, fundamental para o processo de criação e difusão de conhecimento pelo modelo analisado. Mesmo quando ocorre uma atividade de socialização, esta é de forma centralizada nos hubs da rede. Esta conclusão só foi possível graças à aplicação de ferramentas da ARS no modelo SECI. O aprofundamento desta pesquisa, com base nas práticas recomendadas e utilizadas na organização oriundas da ITIL, versão 2, permitirá complementar este estudo, ainda que de forma exploratória e indicativa, revelando possíveis distorções da sobreposição das redes formais (desenhadas pelo ITIL) com as redes informais mapeadas pela ARS. Estas distorções, se confirmadas, representarão pontos críticos para correção e ajustes, reduzindo os riscos e fragilidade das redes.

Para finalizar, é possível ressaltar a importância da pesquisa quando observada sob a visão de oportunidades de melhorias, as quais foram absorvidas e trabalhadas pela organização nos períodos seguintes da sua realização.

\section{Referências}

ALBERT, R.; BARABÁSI, A-L. Statistical mechanics of complex networks. Reviews of Modern Physics, v. 74, n. 1, p. 47-97, 2002. http://dx.doi.org/10.1103/ RevModPhys.74.47

BARABÁSI, A. L.; ALBERT, R. Emergence of Scaling in Random Networks. Science, n. 286, p. 509-512, 1999. PMid:10521342.

BASTOS, V. B.; SANTOS, M. V. Redes sociais informais e compartilhamento de significados sobre mudança organizacional. Revista de Administração de Empresas, v. 47, n. 3, p. 1-13, 2007.

BOCCALETTI, S. et al. Complex networks: Structure and dynamics. Physics Reports, v. 424, n. 4, p. 175-308, 2006. http://dx.doi.org/10.1016/j.physrep.2005.10.009

CLEAN, A. F. Desafios e propostas estratégicas. In: OLIVEIRA, F. B. (Ed.). Tecnologia da informação e da comunicação: desafios e propostas estratégicas para o desenvolvimento dos negócios. São Paulo: Pearson Prentice Hall, 2006. p. 63-69.

COMPUTERWORLD. Apesar de gerar gastos, SarbanesOxley reduziu fraudes. COMPUTERWORLD, 2007. Disponível em:<http://computerworld.uol.com.br/ gestao/2007/07/13/idgnoticia.2007-07-13.5731844639/>. Acesso em: 09 ago. 2010.

COSTA, L. F. et al. Characterization of complex networks: A survey of measurements. Advances in Physics, v. 56, n. 1, p. 167-242, 2007. http://dx.doi. org/10.1080/00018730601170527

DAVENPORT, T. H.; PRUSAK, L. Conhecimento Empresarial: como as organizações gerenciam o seu capital. 4. ed. São Paulo: Elsevier Brazil, 1998. 
FAGUNDES, E. M. COBIT um kit de ferramentas para a excelência na gestão de TI. Tecnologia \& Inovação, 2007. Disponível em:<http://www.efagundes.com/artigos/ COBIT.htm>. Acesso em: 09 ago. 2010.

LÓPEZ, J.; SCOTT, J. Social structure. Open University Press, 2000.

MARTELETO, R. M. Análise de redes sociais: aplicação nos estudos de transferência da informação. Ciência da Informação, v. 30, n. 1, p. 71-81, 2001.

MATHEUS, R. F. Métodos de análise de redes sociais de informação associados a processos organizacionais. 2005. 182 f. Dissertação (Mestrado em Ciência da Informação)-Escola de Ciência da Informação, Universidade Federal de Minas Gerais, Belo Horizonte, 2005.

MATHEUS, R. F.; SILVA, A. B. O. Análise de redes sociais como método para a Ciência da Informação. DataGramaZero-Revista de Ciência da Informação, 2006.

MORENO, J. L. Inter-personal therapy and the psychopathology of inter-personal relations. Sociometry, p. 9-76, 1937. http://dx.doi.org/10.2307/2785258

NEWMAN, M. E. J. The structure and function of complex networks. SIAM review, p. 167-256, 2003. http://dx.doi. org/10.1137/S003614450342480
NONAKA, I.; TAKEUCHI, H. Criação de conhecimento na empresa. Rio de Janeiro: Campus, 1997.

NONAKA, I.; TAKEUCHI, H. Gestão do conhecimento. Tradução de A. Thorell. Porto Alegre: Bookman, 2008.

POLYANI, M. The tacit dimension. reissue. ed. Chicago: University of Chicago Press, 2009.

VALDEUSO, C. Alinhando a TI com o negócio. In: OLIVEIRA, F. B. (Ed.). Tecnologia da informação e da comunicação: desafios e propostas estratégicas para o desenvolvimento dos negócios. São Paulo: Pearson Prentice Hall, 2006. p. 35-42.

VON KROGH, G. et al. Facilitando a criação do conhecimento: reinventando a empresa com o poder da inovação contínua. Rio de Janeiro: Campus, 2001.

WASSERMAN, S.; FAUST, K. Social network analysis: Methods and applications. Cambridge university press, 1999.

WATTS, D. J.; STROGATZ, S. H. Collective dynamics of 'small-world'networks. Nature, v. 393, n. 6684, p. 440-442, 1998. PMid:9623998. http://dx.doi. org/10.1038/30918

WEILL, P.; ROSS, J. W. Governança de TI, tecnologia da informação. São Paulo: M. Books do Brasil, 2006. 